\title{
Rotational modulation, shear, and cyclic activity in HII 1883
}

\author{
J. B. Milingo ${ }^{1}$, S. H. Saar ${ }^{2}$, L. A. Marschall ${ }^{1}$, and J. R. Stauffer ${ }^{3}$ \\ ${ }^{1}$ Department of Physics, Gettysburg College, Gettysburg, PA 17325, USA \\ email: jmilingo@gettysburg.edu, marschal@gettysburg.edu \\ ${ }^{2}$ Harvard-Smithsonian Center for Astrophysics, Cambridge, MA 02138, USA \\ email: saar@head.cfa.harvard.edu \\ ${ }^{3}$ Spitzer Science Center, Pasadena, CA 91125, USA \\ email: stauffer@ipac.caltech.edu
}

\begin{abstract}
We present a 30 year compilation of V-band differential photometry for the Pleiades $\mathrm{K}$ dwarf HII 1883. HII 1883 has an average rotational period $\left\langle P_{r o t}\right\rangle$ of $\sim 0.235 d$ and displays rotational modulation due to non-uniform surface brightness as large as 0.2 magnitudes in $\mathrm{V}$. Preliminary work yields a cycle period of $\sim 9 y r s$ and rotational shear $\delta P_{\text {rot }} /\left\langle P_{\text {rot }}\right\rangle$ considerably less than solar. With such a long baseline of data available we can explore many aspects of the star's photometric variability. We present studies of the variation of the rotational modulation amplitude, $\langle V\rangle$, and $P_{\text {rot }}$ over the cycle.
\end{abstract}

Keywords. Stars: activity, stars: late-type, stars: spots, stars: rotation

\section{Introduction}

Long-term photometry of spotted stars can be exploited to constrain our understanding of the underlying magnetic activity in late-type stars. Tying changes in integrated brightness to periodic changes in spot coverage, a long-running time series of photometry can be used to look for cycle periods analogous to the Sun's. Given that heavily spotted stars show rotational modulation due to overall spot coverage and asymmetry, if a time series is available that exceeds the rotational period of the star then seasons of observations can be examined for changes in the mean brightness of the star as well as changes in the rotation period. The rotational periods of spotted stars are determined from light curves that emerge as a result of the distribution of spots that exist at the time of observation. The period determined in this fashion depends on the latitude of the active regions producing the brightness modulation. Although the time scales associated with the emergence and decay of active regions must be considered here, changes in rotational period can be used as a limited measure of the surface differential rotation (Donahue et al. 1996).

This paper presents a nearly 30 year compilation of $\mathrm{V}$ band photometry for HII 1883 . HII 1883 is one of three stars in a long-term study that Gettysburg College joined in 1992. HII 1883 is a (presumably) single K2 V Pleiades member that shows rotational modulation due to spot coverage (Stauffer 1984a; Stauffer et al. 1984b). With a V magnitude of 12.6 HII 1883 displays modulation as large as 0.2 magnitudes and a very rapid rotation rate. With published photometry dating back to 1980 , the existing time series and continued monitoring of HII 1883 affords us the opportunity to explore various aspects of this active star's photometric variability. 


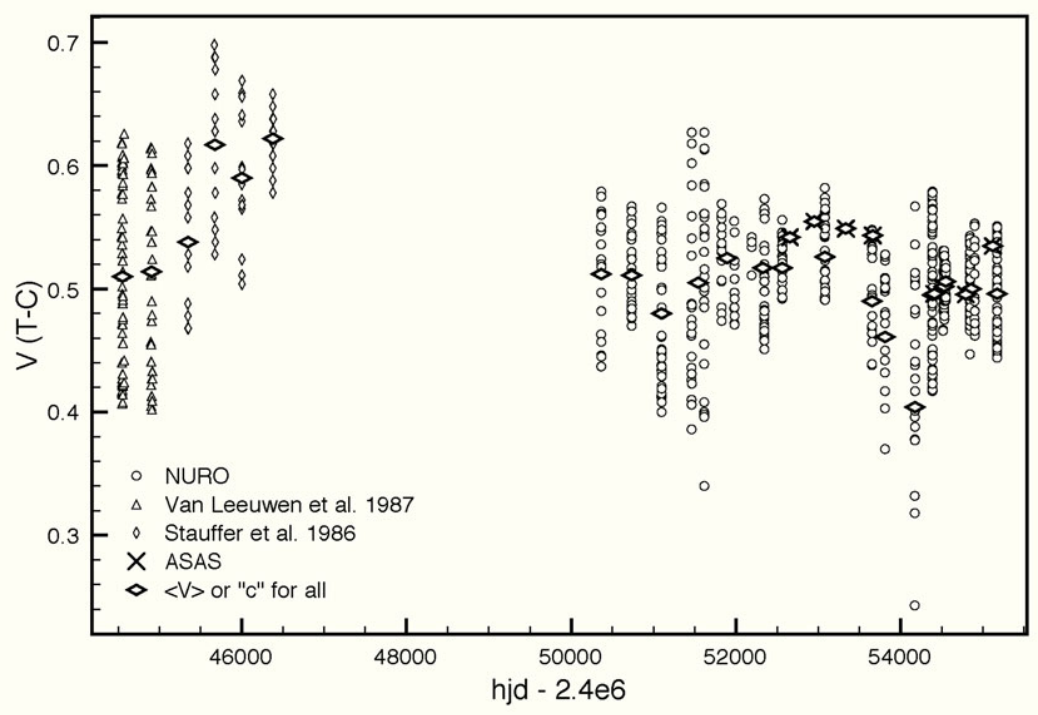

Figure 1. Compiled time series, differential $V_{\text {mag }}$.

\section{Data}

Figure 1 shows the compiled time series. The data from 1992-2009 was acquired using the Lowell Observatory $0.8 \mathrm{~m}$ telescope which, under an agreement with Northern Arizona University and the NURO Consortium, is operated $60 \%$ of the time as the National Undergraduate Research Observatory (NURO). As a NURO consortium member Gettysburg College is allocated 1-2 weeks of dedicated time each year to observe various targets including HII 1883. The NURO data is V band differential photometry using check and comparison stars from earlier studies by John Stauffer (Stauffer et al. 1987). The data from 1980 and 1981 is from Van Leeuwen et al. (1987), and data from the early-mid 1980's is from Stauffer et al. (1986). We have included additional data from the All Sky Automated Survey (ASAS) project (Pojmanski 2002) that stretches from 2003-2009. All data in the series is either standard or differential photometry using the comparison star from the NURO work. Horizontal diamonds in Fig. 1 mark the mean V of each chunk of data. The data from the ASAS project is noisy given the magnitude of HII 1883, hence these runs are simply represented by their mean values.

\section{Analysis}

Before it could be included in the time-series analysis, each run of data was examined to determine whether a light curve could be constructed. Breaks in the data considered the length of each run, how many observation points it contained, and possible contributions from the emergence and decay of different active regions. A value of $\langle V\rangle$ was determined for each chunk of data either by directly calculating the mean or by applying a fit to the phased light curve then taking the mean of that fit. This was done to mitigate any odd windowing of the observations.

A cycle period was determined by running a Lomb-Scargle (L-S) analysis on the $\langle V\rangle$ values. An initial rough analysis including just the Van Leeuwen and NURO data produced $P_{c y c}=7.7 y r s$ with a L-S $F A P=0.1315$. After a more careful examination of 
the NURO data and including the Stauffer and ASAS data we find $P_{c y c}=9.1 y r s$ with a L-S FAP $=0.0215$. We point out that the Stauffer data is somewhat shifted from both the NURO and Van Leeuwen data. In this analysis we have applied an offset to account for the different photometric data sets. Although this optimized our $P_{c y c}$ value, we are keenly aware of the uncertainty this introduces. We continue to investigate the nature of this offset and the existence of unpublished data that could fill the large gap in observations.

Figure 2 shows phased light curves from (left to right) November 1980 (Van Leeuwen et al. 1987) and NURO data from October 2007 and December 2009. All three are phased with the same $T_{0}$ using the dominant period determined from a L-S analysis of each run (y-axes are not on the same scale). The dominant periods for each of these light curves are (a) $P_{\text {rot }}=0.2355 \pm 0.0007 d$ (b) $P_{\text {rot }}=0.234 \pm 0.004 d$ and (c) $P_{\text {rot }}=0.236 \pm 0.004 d$; $F A P<10^{-3}$ in each case.

Figure 3 is a plot of the rotational periods for each chunk of data that informed the cycle period. Considering the uncertainty in these values we see no clear pattern in $P_{\text {rot }}$ emerging over the time series (or when phased with $P_{c y c}$ ). Including all values we find $\left\langle P_{\text {rot }}\right\rangle=0.2352 \pm 0.0014 d$ (standard deviation). Adopting $P_{\max }-P_{\min }$ to determine $\delta P_{\text {rot }}$ we find $\delta P_{\text {rot }} /\left\langle P_{\text {rot }}\right\rangle \leqslant 0.04$. Eliminating the highest and lowest values as possible outliers we find $\left\langle P_{\text {rot }}\right\rangle=0.2352 \pm 0.0006 d$ and $\delta P_{\text {rot }} /\left\langle P_{\text {rot }}\right\rangle \leqslant 0.01$. We present both as upper limits considering the errors in $P_{\text {rot }}$ overlap to a degree that makes our detection uncertain. Regardless our measure of differential rotation is considerably less than the solar value, measured as a star in Ca II HK, of 0.15 (Donahue et al. 1996).

Using the light curves from each data chunk, we examined the rotational modulation amplitudes as an alternate proxy, albeit muddy, for activity. Running a L-S analysis on the Van Leeuwen, Stauffer, and NURO data sets (no ASAS) we found $P_{c y c} \sim 2900 d$ with a $F A P=0.171$, only a weak indication of periodicity. There was a clear phase shift amongst the rotational light curves (see Figure 2). With all light curves phased to the same $T_{0}$ we ran a L-S analysis on the phase shifts and found $P_{c y c} \sim 2800 d$ with a $F A P=0.016$. With very little deviation in the $P_{\text {rot }}$ values we interpret these phase shifts to be the march of active longitudes at play, but small changes in $P_{\text {rot }}$ are tangled in here complicating the issue. With such a small spread in $P_{\text {rot }}$ the strength of the dominant periodicity in the phase shifts is intriguing.
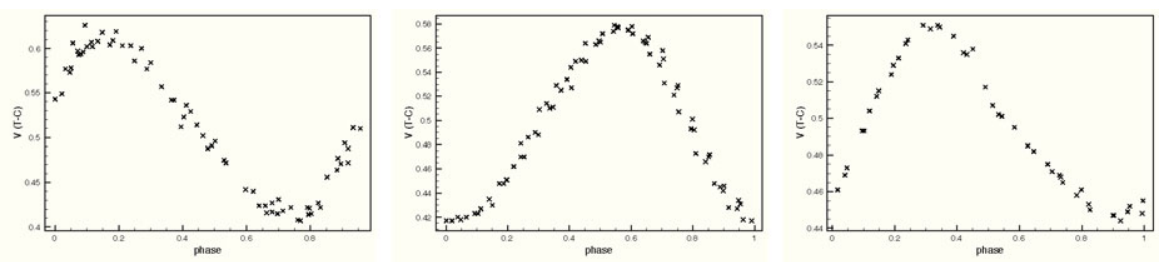

Figure 2. Light curves phased to the same $T_{0}$.

\section{Future Work}

Our work continues with HII 1883, as well as the other two Pleiades stars in this study (which are also rapid rotators). Continued monitoring at NURO and the addition of complimentary data, both archival (Krishnamurthi et al. (1998) for example) and 


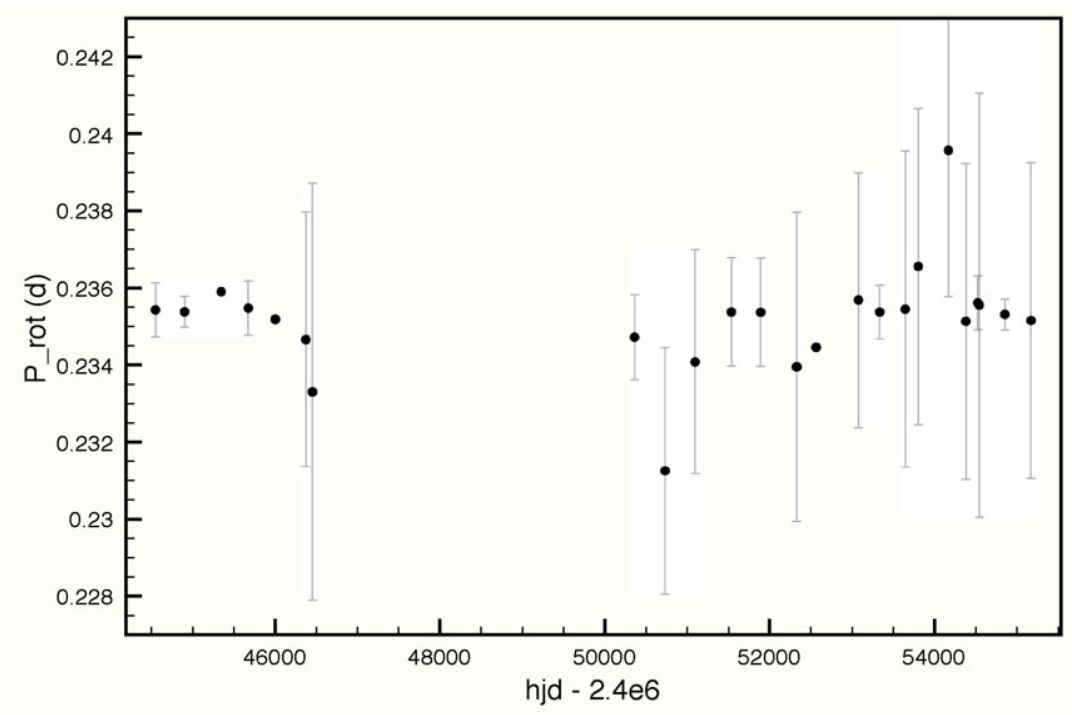

Figure 3. $P_{\text {rot }}$ values throughout the time series.

newly acquired, will help paint a clearer picture of this active star. Many thanks to all, particularly Dr. Dana Backman, who have helped to move this project forward.

\section{References}

Donahue, R. A., Saar, S. H., \& Baliunas, S. L. 1996, Astrophys. J., 466, 384

Krishnamurthi, A., Terndrup, D. M., Pinsonneault, M. H., Sellgren, K., Stauffer, J. R., Schild, R., Backman, D. E., Beisser, K. B., Dahari, D. B., Dasgupta, A., Hagelgans, J. T., Seeds, M. A., Anand, R., Laaksonen, B. D., Marschall, L. A., \& Ramseyer, T. 1998, Astrophys. J., 493, 914

Pojmanski, G. 2002, AcA, 52, 397

Stauffer, J. R., Schild, R. A., Baliunas, S. L., \& Africano, J. L. 1987, Pub. Astron. Soc. Pac., 99,471

Stauffer, J. R., Dorren, J. D., \& Africano, J. L. 1986, AJ, 91, 1443

Stauffer, J. R. 1984, Astrophys. J., 280, 189

Stauffer, J. R., Hartmann, L., Soderblom, D. R., \& Burnham, N. 1984, Astrophys. J., 280, 202

Van Leeuwen, F., Alphenaar, P., \& Meys, J. J. M. 1987, A\&AS, 67, 483 